\title{
Extrapleural pneumonectomy followed by intracavitary intraoperative hyperthermic cisplatin with pharmacologic cytoprotection for treatment of malignant pleural mesothelioma: A phase II prospective study
}

Tamara R. Tilleman, MD, PhD, ${ }^{a}$ William G. Richards, PhD, ${ }^{a}$ Lambros Zellos, MD, MPH, ${ }^{a}$

Bruce E. Johnson, MD, ${ }^{\mathrm{b}}$ Michael T. Jaklitsch, MD, ${ }^{\mathrm{a}}$ Jordan Mueller, ${ }^{\mathrm{a}}$ Beow Yong Yeap, $\mathrm{ScD},{ }^{\mathrm{c}}$

Aneil A. Mujoomdar, MD, ${ }^{\mathrm{a}}$ Christopher T. Ducko, MD, ${ }^{\mathrm{a}}$ Raphael Bueno, MD, ${ }^{\mathrm{a}}$ and

David J. Sugarbaker, MD

Objective: We sought to prospectively determine the feasibility and safety of hyperthermic intraoperative intracavitary cisplatin perfusion immediately after extrapleural pneumonectomy in the treatment of malignant pleural mesothelioma.

Methods: Patients with malignant pleural mesothelioma who were surgical candidates underwent extrapleural pneumonectomy followed by hyperthermic intraoperative intracavitary cisplatin perfusion, consisting of a 1-hour lavage of the chest and abdomen with cisplatin $\left(42^{\circ} \mathrm{C}\right)$ at $225 \mathrm{mg} / \mathrm{m}^{2}$. Pharmacologic cytoprotection consisted of intravenous sodium thiosulfate with or without amifostine. Morbidity and mortality were recorded prospectively.

Results: Ninety-six (79\%) of 121 enrolled patients underwent extrapleural pneumonectomy, of whom 92 (76\%) received hyperthermic intraoperative intracavitary cisplatin perfusion after extrapleural pneumonectomy. Fiftythree $(58 \%)$ patients had epithelial tumors, and $39(42 \%)$ had nonepithelial histology. Hospital mortality was $4.3 \%$. Morbidity (grade 3 or $4,49 \%$ ) included atrial fibrillation in $22(23.9 \%)$ patients, venous thrombosis in $12(13 \%)$ patients, and laryngeal nerve dysfunction in $10(11 \%)$ patients. Nine patients had renal toxicity, which was attributable to cisplatin in 8 of them. Among the 27 patients who also received amifostine $\left(910 \mathrm{mg} / \mathrm{m}^{2}\right)$, 1 patient had grade 3 renal toxicity attributable to cisplatin. Recurrence of malignant pleural mesothelioma was documented in $47(51 \%)$ patients, with ipsilateral recurrence in $17.4 \%$ of patients. The median survival of the 121 enrolled patients was 12.8 months.

Conclusions: Hyperthermic intraoperative intracavitary cisplatin perfusion following extrapleural pneumonectomy can be performed with acceptable morbidity and mortality. The use of amifostine in addition to sodium thiosulfate might reduce cisplatin-associated renal toxicity. Hyperthermic intraoperative intracavitary cisplatin perfusion following extrapleural pneumonectomy might enhance local control in the chest.

Malignant pleural mesothelioma (MPM) is a rare thoracic cancer that originates in pleural mesothelial cells and progresses relentlessly to encase the lungs and mediastinum, ${ }^{1}$ ultimately causing death by locoregional extension. This malignancy is usually associated with previous asbestos exposure $^{2}$ and is refractory to standard treatment modalities, including chemotherapy and radiation therapy. As a consequence, patients with MPM usually have a poor prognosis,

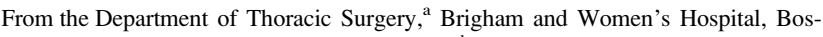
ton, Mass; the Dana Farber Cancer Institute ${ }^{b}$; and the Department of Medicine, ${ }^{c}$ Massachusetts General Hospital, Boston, Mass.

Read at the Eighty-eighth Annual Meeting of The American Association for Thoracic Surgery, San Diego, Calif, May 10-14, 2008.

Received for publication May 15, 2008; revisions received Jan 9, 2009; accepted for publication Feb 23, 2009

Address for reprints: David J. Sugarbaker, MD, Division of Thoracic Surgery, Brigham and Women's Hospital, 75 Francis Street, Boston, MA 02115 (E-mail: dsugarbaker@partners.org).

J Thorac Cardiovasc Surg 2009;138:405-11

0022-5223/\$36.00

Copyright (c) 2009 by The American Association for Thoracic Surgery

doi:10.1016/j.jtcvs.2009.02.046
}

and the median survival from the time of presentation to death is in the range of 7 to 12 months. $^{3}$

Multimodality therapy, which includes maximal cytoreductive surgery ${ }^{4}$ followed by radiotherapy for local control and chemotherapy for both locoregional and systemic control, has resulted in prolonged survival in selected patients who have relatively early disease and adequate cardiopulmonary function. The surgical treatment of MPM was initially limited by unacceptable morbidity and mortality for complete resection. ${ }^{5,6}$ The most effective surgical approach for resection of macroscopic disease is extrapleural pneumonectomy (EPP) ${ }^{7-9}$ Patients who are not candidates for EPP as a result of insufficient cardiopulmonary reserve, advanced age, or disease distribution might be candidates for cytoreduction with pleurectomy/decortication $(\mathrm{P} / \mathrm{D}) .{ }^{10}$ During the past 2 decades, the safety of these 2 effective cytoreductive procedures was established in several centers of expertise worldwide, providing surgeons with the ability to achieve macroscopic complete resection $(\mathrm{MCR})^{4}$ with acceptable morbidity and mortality. In combination with a 


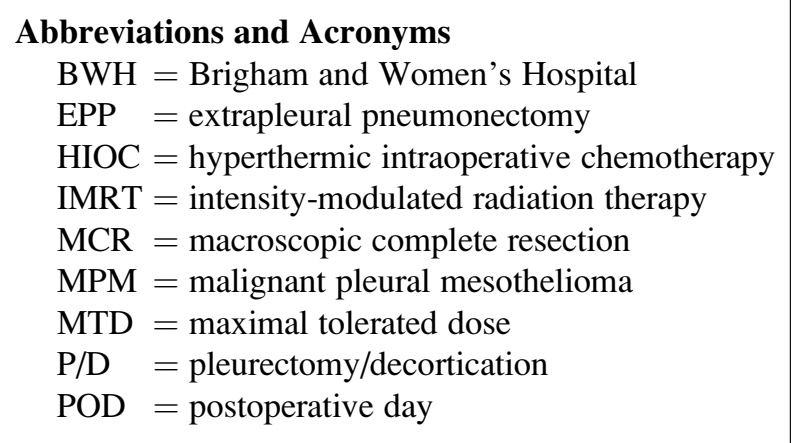

variety of adjuvant therapies, primary surgical intervention has led to multiple reports of prolonged disease-free interval and survival relative to historical and contemporary single-modality treatment strategies. ${ }^{11-13}$ Despite these advances, however, most patients experience eventual recurrence of their mesothelioma and death.

MPM exhibits a progression pattern characterized by a tendency to recur locally. One study examining the patterns of recurrence showed that $54 \%$ of the 25 patients who had recurrence after trimodality therapy, the predominant site of first failure was within the ipsilateral hemithorax ( $67 \%$ of all recurrences) followed by the abdomen $(50 \%){ }^{14}$ The mechanism of this recurrence pattern might be regrowth of microscopic residual or shed tumor cells or a second primary disease arising in the peritoneum or on the pleural surface. The local nature of disease recurrence has emphasized the need for improved locoregional control, and trials of intraoperative radiation ${ }^{15}$ and photodynamic therapy ${ }^{16}$ have been undertaken. Potential shortfalls of these strategies, however, include the inability to treat intra-abdominal or peritoneal sites of disease.

We therefore investigated hyperthermic intraoperative chemotherapy (HIOC) lavage as a means of delivering high-dose regional therapy simultaneously to the 2 body cavities at highest risk for recurrence with the objective of sterilizing the microscopic surgical margins. Phase I studies with bicavitary (thorax and abdomen) cisplatin perfusion after $\mathrm{EPP}^{9,17}$ or $\mathrm{P} / \mathrm{D}^{10}$ have been reported. These initial studies established a maximal tolerated dose (MTD) for cisplatin, confirmed its safety, and suggested the efficacy of the treatment in terms of improved time to disease progression and survival. In the present prospective phase II trial, we sought to determine the feasibility and associated mortality and morbidity of administering HIOC at the established MTD in a consecutive series of patients undergoing EPP.

\section{MATERIALS AND METHODS Objectives}

The primary objectives of this study were to determine the feasibility of HIOC with cisplatin immediately after EPP and to document the morbidity and mortality of this treatment with particular reference to renal function.
The treatment protocol consisted of EPP followed immediately by intrathoracic/intraperitoneal cisplatin HIOC at a dose of $225 \mathrm{mg} / \mathrm{m}^{2}$, with cytoprotection (mainly to prevent nephrotoxicity) using intravenous sodium thiosulfate with or without intravenous amifostine. ${ }^{18}$ The secondary objectives were to evaluate the pattern and timing of disease recurrence and patient survival.

\section{Eligibility and Enrollment}

This prospective phase II protocol was approved by the institutional review board. Patients with biopsy-proved MPM who were candidates for $\mathrm{EPP}^{19}$ were offered participation in this study, and informed consent was obtained from the patients. Study-specific inclusion criteria included the following: pathologic diagnosis confirmed by the institutional mesothelioma pathology review panel, absence of radiographic evidence of metastatic disease, adequate cardiopulmonary function (ie, ejection fraction $>45 \%$, absence of severe cardiac valvular abnormalities, absence of pulmonary hypertension determined by using echocardiographic analysis, and preoperative $\mathrm{FEV}_{1}$ of $\geq 2 \mathrm{~L}$ and predicted postoperative $\mathrm{FEV}_{1}$ of $>0.8 \mathrm{~L}$ based on a quantitative ventilation-perfusion scan), adequate renal function (ie, serum creatinine level of $<1.5 \mathrm{mg} / \mathrm{dL}$ and estimated creatinine clearance of $>45$ $\mathrm{mL} / \mathrm{min}$ ), adequate hepatic function (aspartate aminotransferase level of $<80 \mathrm{IU} / \mathrm{L}$ and total bilirubin level of $<1.9 \mathrm{mg} / \mathrm{dL}$ ), normal contralateral pulmonary function, and Karnofsky performance status of 70 or greater. Exclusion criteria included the following: previous chemotherapy, radiation therapy, or both for mesothelioma; any chemotherapy, radiation therapy, or both administered within 3 years for another malignancy; prior malignancy with remission for less than 3 years; pregnancy at the time of the operation; or any severe nonmalignant comorbid disease.

\section{Surgical Intervention and Intraoperative Hyperthermic Lavage}

EPP was attempted for all enrolled patients. This operation consists of en bloc resection of the visceral, parietal, and mediastinal pleurae; the lung; the ipsilateral diaphragm; and the portion of the pericardium that borders the ipsilateral lung, as previously described. ${ }^{19}$ The pericardial and hemidiaphragmatic defects are reconstructed with a polytetrafluoroethylene patch (Gore-Tex patch; W. L. Gore \& Associates, Flagstaff, Ariz). Patients in whom MCR $\left(<1.0 \mathrm{~cm}^{3}\right.$ tumor) could not be achieved were considered unresectable.

After extirpation of the entire specimen, careful attention was directed toward complete hemostasis of the surgical field. Intracavitary lavage of the ipsilateral chest and abdominal cavities was carried out for 60 minutes with a solution of $225 \mathrm{mg} / \mathrm{m}^{2}$ cisplatin in dialysate (Baxter, Deerfield, Ill) maintained at $42^{\circ} \mathrm{C}$, as previously described. ${ }^{10}$ Intravenous sodium thiosulfate for renal protection $\left(4 \mathrm{~g} / \mathrm{m}^{2}\right.$ bolus in $250 \mathrm{~mL}$ of sterile water over 10 minutes) was administered immediately after the cisplatin lavage was concluded. This was followed by an additional infusion of $12 \mathrm{~g} / \mathrm{m}^{2}$ sodium thiosulfate in $250 \mathrm{~mL}$ of sterile water over 6 hours. Eighty-two of 92 patients received a second identical infusion of sodium thiosulfate. ${ }^{20}$ After interim analysis of renal toxicity in 65 patients showing that 7 had grade 4 renal toxicity, the protocol was amended, and 27 patients also received amifostine for additional renal protection. The infusion of $910 \mathrm{mg} / \mathrm{m}^{2}$ amifostine (Ethyol; Alza Pharmaceuticals, Mountain View, Calif) was administered intravenously over 15 minutes, starting 30 minutes before cisplatin perfusion and 90 minutes before sodium thiosulfate administration. ${ }^{21}$

The temperature of the perfusate was monitored continuously with intrathoracic and intra-abdominal probes, and the body temperature was monitored with an esophageal probe. Furosemide, low-dose dopamine, mannitol, and/or intravenous fluids were used to maintain urine output at greater than $100 \mathrm{~mL} / \mathrm{h}$ for the duration of the lavage and for 1 hour after its completion. Patients were maintained on positive pressure ventilation for 24 hours postoperatively to maximize lung expansion. 


\section{Post-treatment Follow-up}

After the operation, complete blood counts and chemistry panels were monitored daily until discharge. Adverse events that occurred during postoperative hospitalization were graded according to Common Terminology Criteria for Adverse Events version 3.0 (https://webapps.ctep.nci.nih.gov/ webobjs/ctc/webhelp/welcome_to_ctcae.htm). After discharge, patients were seen and evaluated by a physician in ambulatory follow-up at 1 to 2 weeks, 6 to 8 weeks, and then 4-month intervals. A computed tomographic scan of the chest was obtained at the second follow-up and every 4 months thereafter to monitor for evidence of recurrence. Date of recurrence was considered to be the date of the first radiographic study during which recurrence was demonstrable. Mortality was considered to be disease related. Adjuvant therapy was neither mandated nor prohibited by the protocol, and most of the patients were treated outside the Boston area. Therefore data regarding adjuvant therapy were not collected and are not reported herein.

\section{Statistical Analysis}

Morbidity was defined as any adverse event occurring during the postoperative hospitalization. Mortality was defined as any death during the initial postoperative hospitalization or within 30 days of the operation. Pathologic staging was assigned for all cases by using American Joint Committee on Cancer and Brigham and Women's Hospital (BWH) criteria. ${ }^{22}$ Overall survival was estimated by using the Kaplan-Meier method, and the log-rank test was used to compare differences between patient subgroups. Cancer-specific survival was based on the cumulative incidence estimate, with death associated with intensity-modulated radiation therapy (IMRT; 8 patients) considered as a competing risk. ${ }^{23}$ The Gray test was used to compare the cancer-specific survival difference between patient subgroups. ${ }^{24}$ The analysis of overall survival was computed with SAS version 9.1 (SAS Institute, Inc, Cary, NC) statistical software, whereas the competing-risks analysis was performed with the cmprsk software package in $\mathrm{R}$ version 2.5.1. Contingency data analysis was conducted by using the Fisher's exact test. The sample size was chosen to provide the $85 \%$ statistical power needed to determine whether the addition of HIOC is associated with an improvement in overall survival compared with that seen in historical control subjects who underwent EPP without HIOC at our institution. The goal was to detect a hazard ratio of 0.714 at a 1 -sided significance level of $10 \%$, which is similar to the survival gain observed among the P/D cohort that underwent HIOC. ${ }^{25}$ One hundred twenty-one patients were enrolled to ensure an effective sample size of at least 85 evaluable patients who actually completed HIOC. The patient numbers also provided adequate precision for the estimation of mortality, morbidity, and toxicity. Although a priori hypotheses were not planned for the secondary end points, the study design included an early stopping rule for unacceptable mortality.

\section{RESULTS}

\section{Enrollment and Treatment}

One hundred twenty-one patients were enrolled in the study over a $2 \frac{1}{2}$-year period (January 2004 to June 2006). The study cohort was generally representative of patients with MPM presenting for surgical therapy in terms of demographic and histologic characteristics but included a relatively high percentage of patients with stage III disease or greater (Table 1). Twenty-five patients had tumors that were not resectable by means of EPP because of invasion into the chest wall $(n=21)$, invasion into major central blood vessels $(n=3)$, or diffuse abdominal metastases noted at the time of the operation $(n=1)$. Of the patients whose tumors were deemed unresectable by means of EPP, 14
TABLE 1. Overview of enrolled patients treated by using EPP with HIOC

\begin{tabular}{lrc}
\hline \multicolumn{1}{c}{ Patients enrolled } & No. & Percentage of 121 \\
\hline General & & \\
Enrolled & 121 & \\
Resected & 96 & 79 \\
Treated & 92 & 76 \\
Partial resection or P/D & 14 & 12 \\
Unresectable & 11 & 9 \\
EPP with no HIOC & 4 & 3
\end{tabular}

Patients treated

Percentage of 92

Sex

$\begin{array}{lll}\text { Male } & 73 & 79 \\ \text { Female } & 19 & 21\end{array}$

Age (y)

Median (range) $60(27-78)$

Laterality

Right side $42 \quad 46$

Left side $\quad 50 \quad 54$

Tumor histology

Epithelial

Biphasic

Sarcomatoid

AJCC staging

I

II

III

IV

50

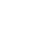

$36-39$

$3 \quad 3$

BWH staging

I 6

II 28

III

Median (range)

79

21

Hospital stay

6

58

39

3

2

$12 \quad 13$

$50 \quad 54$

$28 \quad 30$

$\overline{E P P}$, Extrapleural pneumonectomy; HIOC, hyperthermic intraoperative chemotherapy; $P / D$, pleurectomy/decortication; $A J C C$, American Joint Committee on Cancer; $B W H$, Brigham and Women's Hospital.

underwent partial tumor debulking, and no resection was attempted for the remaining 11 patients.

The majority of the patients (96 [79\%]) were found to have resectable tumors and underwent EPP. Of these, 92 patients completed HIOC treatment according to the protocol. Four patients did not complete HIOC treatment because of hemodynamic instability $(n=3)$ or technical failure of the perfusion system $(n=1)$. These patients were excluded from the study, yielding a study cohort of 92 patients.

\section{Morbidity and Mortality}

Mortality. Four of the 92 treated patients died (mortality, $4.3 \%$ ). One patient died within 30 days of the operation from multiorgan failure after cardiopulmonary arrest (postoperative day [POD] 24). Three additional patients died during the postoperative hospitalization period (multiorgan 
TABLE 2. Grade 3 and 4 adverse events

\begin{tabular}{lcccc}
\hline & Grade & Grade & Total & Percentage \\
& $\mathbf{3}$ & $\mathbf{4}$ & patients & of 92 \\
\hline Adverse event & & & & \\
$\quad$ AF & 17 & 5 & 22 & 23.9 \\
Major morbidity (non-AF) & & & & \\
Thrombosis/thrombus/embolism & 8 & 4 & 12 & 13.0 \\
Laryngeal nerve dysfunction & 10 & & 10 & 10.9 \\
ARDS/respiratory failure & 1 & 5 & 6 & 6.5 \\
Cardiac ischemia or CP arrest & 3 & 2 & 5 & 5.4 \\
Chylothorax & 4 & & 4 & 4.3 \\
Increased creatinine & 6 & 3 & 9 & 9.8 \\
Renal failure & & 3 & 3 & 3.3 \\
Pneumonia & 3 & & 3 & 3.3 \\
Fistula, bronchus & 2 & 1 & 3 & 3.3 \\
Empyema & 1 & 1 & 2 & 2.2 \\
Ileus & 2 & & 2 & 2.2 \\
Intraoperative injury, carotid & 1 & & 1 & 1.1 \\
$\quad$ artery & & & & 1 \\
Fistula, enteric & & & & \\
Intraoperative injury, spleen & 1 & & & 1 \\
\hline$A F$, Atrial fibrillation; $A R D S$, acute respiratory distress syndrome; $C P$, cardiopulmo- \\
nary.
\end{tabular}

failure at POD 43, sepsis after bronchopleural fistula at POD 53, and sepsis after patch dehiscence at POD 160).

Morbidity. Forty-five patients had postoperative complications (48.9\% morbidity, Table 2$)$. The most common complication was atrial arrhythmia (22 [24\%] patients). Grade 3 to 4 renal toxicity occurred in 9 patients, manifesting as increased serum creatinine values (9 $[9.8 \%]$ patients) and renal failure (3 $[3 \%]$ patients). The 3 patients who experienced renal failure required dialysis, one for 3 weeks, one for 6 weeks, and one for 7 months. One patient had renal failure attributable to sepsis and multiorgan failure after a perioperative cardiopulmonary arrest. Renal toxicity among the remaining $8(9 \%)$ patients was attributed to cisplatin. Seven of 65 patients treated with sodium thiosulfate alone experienced cisplatin-related renal toxicity compared with only 1 of 27 patients treated with amifostine and sodium thiosulfate $(P=.429$, Table 3$)$.

Recurrence. Radiographic or pathologically confirmed recurrence of MPM was observed in $47(51.1 \%)$ patients. The most common sites of recurrence were the contralateral hemithorax $(61.7 \%$ of all recurrences, 29 [31.5\%] treated patients), abdomen (51.1\% of all recurrences, 24 [26.1\%] treated patients), ipsilateral hemithorax $(34.0 \%$ of all recurrences, $16[17.4 \%]$ treated patients), and distant regions (8.5\% of all recurrences, 4 [4.35\%] treated patients). Two $(3.5 \%)$ of 47 evaluable patients had recurrences only in the ipsilateral hemithorax. The median estimated diseasefree interval for all 92 patients was 15.3 months. Estimated median survival from time of recurrence to death for the 47 patients was 3.1 months.
TABLE 3. Distribution of cisplatin-related renal toxicity in patients treated with and without amifostine $(P=.429)$

\begin{tabular}{lcc}
\hline & Renal toxicity & No renal toxicity \\
\hline With amifostine $(\mathrm{n}=27)$ & 1 & 26 \\
Without amifostine $(\mathrm{n}=65)$ & 7 & 58 \\
\hline
\end{tabular}

Survival. At the time of this analysis, 20 of the 92 treated patients were known to be alive, with a median followup of 31.2 months (range, 16.7-45.8 months). Of the 20 patients who are alive, 15 are without recurrence, and 5 have evidence of recurrence. No patients died of renal toxicity. Forty-five of 92 treated patients died of known recurrence.

Overall median survival of the 121 patients enrolled in this study was 12.8 months. Median survival of the treatment cohort (92 patients) was 13.1 months versus 11.0 months for patients who did not undergo the protocol-specified treatment ( $\mathrm{n}=29, P=.0133$, Figure 1). Patients with epithelial histology $(n=53)$ had a longer overall median survival time (17.1 months) compared with patients with the sarcomatoid/ biphasic cell type ( $\mathrm{n}=39,10.9$ months, $P=.007)$. Earlystage disease (BWH stage I or II, $n=58$ ) was associated with longer median survival times (21.3 months) than advanced-stage disease (BWH stage III; $\mathrm{n}=34,11.5$ months; $P=.0071)$. There were no significant differences between tumor site, sex, or age (Figure 1). According to our analysis, patients with stage III disease had nearly half the survival of patients with stage I and II disease (21.3 vs 11.5 months, $P=.0071)$. This is also less than the overall survival of the treatment cohort (13.1 months, statistical comparison not done). Overall cancer-specific survival was 16.9 months for the treatment cohort $(\mathrm{n}=92)$.

\section{DISCUSSION}

This prospective phase II study establishes the safety and feasibility of administering HIOC after EPP. Of 121 patients, 96 underwent EPP (79\% resectability). Ninety-two of those patients underwent HIOC after EPP. Postoperative mortality was $4.3 \%$. These rates are similar to those previously reported in other EPP series, which report postoperative mortalities between $3.2 \%$ and $7 \% .^{7,8,25,26}$ Forty-five $(48.9 \%)$ of 92 patients experienced either grade 3 or 4 toxicity, which is similar to previous reports. ${ }^{7,11}$ Atrial fibrillation and respiratory adverse events have been previously described as common complications after EPP and occurred at the expected rates. Grade 3 or 4 renal toxicity occurred in $9(9.8 \%)$ patients.

Despite careful screening and perioperative management, renal toxicity attributable to HIOC with cisplatin remains a concern for a small subset of patients. Comparison of the amifostine and sodium thiosulfate group with the sodium thiosulfate-only group suggested a qualitative reduction in the incidence and severity of renal toxicity in the group 

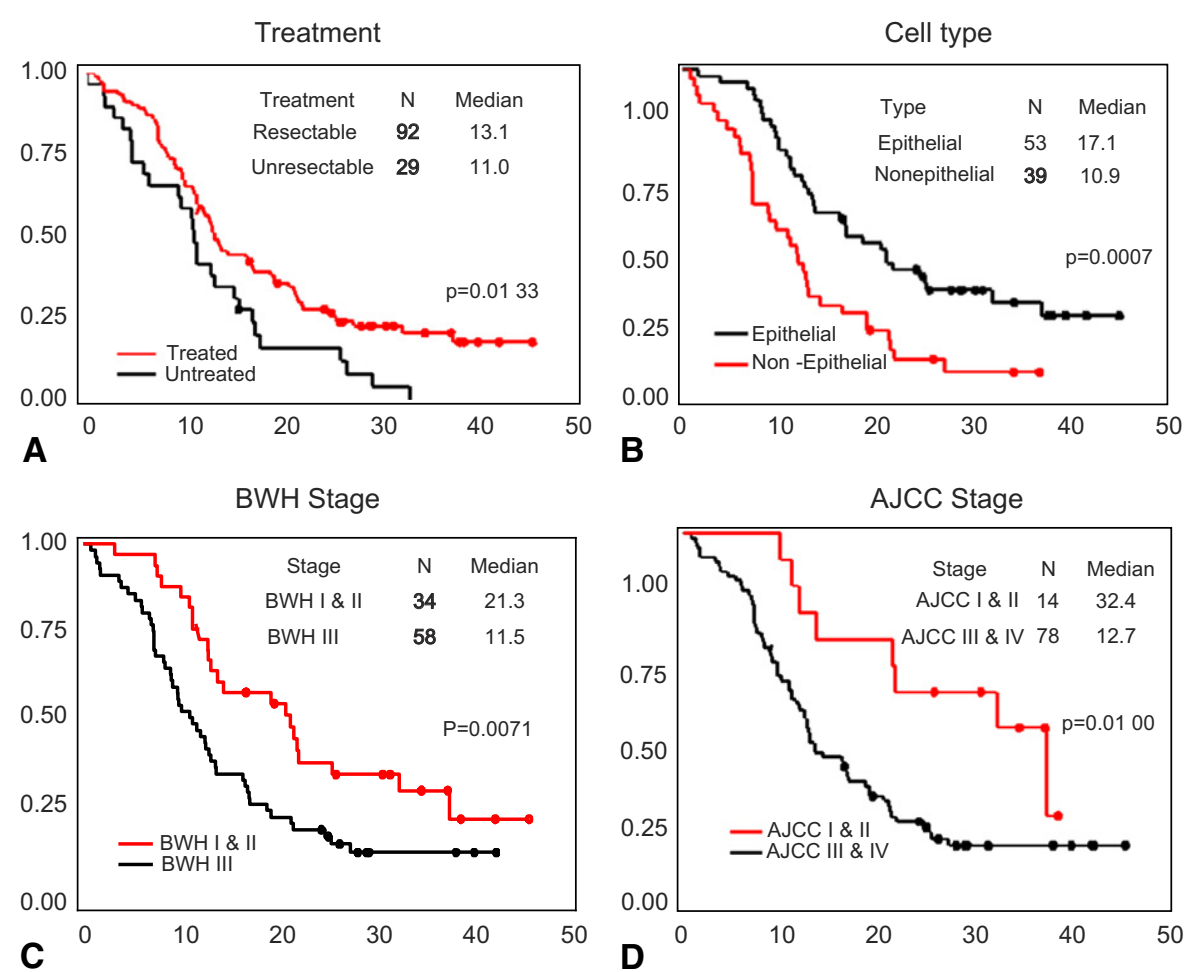

FIGURE 1. Kaplan-Meier survival estimates for all enrolled patients (A) and patients undergoing extrapleural pneumonectomy and hyperthermic intraoperative intracavitary cisplatin lavage (B-C). A, Log-rank comparison demonstrated longer survival for resected patients versus patients who were unresected/ untreated with HIOC. B, Significantly longer survival was demonstrated for patients with epithelial versus nonepithelial tumors. C and D, Pathologic tumor staging by using Brigham and Women's Hospital $(B W H)$ staging and by using the American Joint Committee on Cancer (AJCC) staging system.

receiving both cytoprotective drugs (1/27 compared with $7 / 65$ ). Portions of this work have been presented in abstract form. ${ }^{27}$ This finding was not statistically significant because of the insufficient number of patients in these subgroups, but given the encouraging reduction in renal toxicity when sodium thiosulfate alone (7/65) was replaced with sodium thiosulfate plus amifostine (1/27), we recommend both drugs for renal protection during HIOC with cisplatin. An ongoing investigation is focused on further improvement in the early detection and prevention of renal toxicity.

The median survival of 13.1 months in this treatment cohort (92 patients) is encouraging, given that most had at least stage III disease and nearly half had nonepithelial histology. An exploratory analysis of patients who died of unanticipated complications from HIOC in combination with IMRT revealed a potential median survival of 16.9 months. On the basis of this experience, we do not recommend IMRT in patients with mesothelioma until further research on the safety of this modality can be performed. Patients not treated with EPP received other treatments, including pleurectomy $(\mathrm{n}=$ $14)$ and chemotherapy $(n=15)$. Median survival for patients who did not undergo the above protocol was 11 months.

The observed patterns of disease recurrence ${ }^{14}$ included a low incidence of initial recurrence in the ipsilateral hemithorax $(34.0 \%)$ and a concomitant high incidence of recur- rence in the contralateral hemithorax $(67 \%)$ and abdomen $(50 \%)$, which remains a fatal problem for patients who have undergone pneumonectomy and highlights the need for improved systemic therapies for MPM once local control has been achieved (Table 4).

In this phase II study the safety and feasibility of HIOC after MCR by surgical intervention has been demonstrated as a novel multimodality platform for MPM. Although the increased local dose level of cisplatin permitted by regional application would be expected to improve its efficacy, it remains relatively inactive as a single agent. ${ }^{28-30}$ Response rates for cisplatin alone were reported as $13.6 \%$ and $16.7 \%,{ }^{29,30}$ and a $41.3 \%$ response rate was reported for pemetrexed/cisplatin. ${ }^{30}$ Encouraged by the evidence that combined chemotherapy with pemetrexed plus cisplatin

TABLE 4. Sites of pleural mesothelioma recurrence

\begin{tabular}{|c|c|c|c|}
\hline Site & No. & $\begin{array}{c}\text { Recurrences }(\%) \text {, } \\
\text { Tilleman and } \\
\text { coworkers, 2009* }\end{array}$ & $\begin{array}{c}\text { Recurrences }(\%) \text {, } \\
\text { Baldini and } \\
\text { coworkers, } 1997^{14}\end{array}$ \\
\hline Ipsilateral hemithorax & 16 & 34 & 67 \\
\hline Contralateral hemithorax & 29 & 62 & 33 \\
\hline Abdomen & 24 & 51 & 50 \\
\hline Distant & 4 & 9 & 8 \\
\hline
\end{tabular}

*Current article. 
compared with cisplatin alone for mesothelioma improves survival, ${ }^{30}$ we are currently studying a combination regimen using this alternative. A phase I trial of HIOC with cisplatin at its established MTD in combination with dose-escalated gemcitabine after EPP or $\mathrm{P} / \mathrm{D}$, as appropriate, is currently open at our institution (http://www.clinicaltrials.gov/ct2/ results?term=tilleman). We are using gemcitabine combined with cisplatin rather than pemetrexed initially because it has been safely provided as intracavitary therapy in the abdomen for ovarian cancer.

The present prospective phase II study establishes that HIOC after EPP can be performed with acceptable morbidity and mortality compared with previous studies that report similar numbers. ${ }^{7,25,26}$ Cytoprotection with amifostine and sodium thiosulfate merits further investigation for control of cisplatin-related renal toxicity.

\section{References}

1. Metintas M, Ucgun I, Elbek O, Erginel S, Metintas S, Kolsuz M, et al. Computed tomography features in malignant pleural mesothelioma and other commonly seen pleural diseases. Eur J Radiol. 2002;41:1-9.

2. Tossavainen A. Global use of asbestos and the incidence of mesothelioma. Int J Occup Environ Health. 2004;10:22-5.

3. Goudar RK. New therapeutic options for mesothelioma. Curr Oncol Rep. 2005; 7: 260-5.

4. Sugarbaker DJ. Macroscopic complete resection: the goal of primary surgery in multimodality therapy for pleural mesothelioma. J Thorac Oncol. 2006;1:175-6.

5. Worn H. Chances and results of surgery of malignant mesothelioma of the pleura. Thoraxchir Vask Chir. 1974;22:391-3.

6. Butchart EG, Ashcroft T, Barnsley WC, Holden MP. Pleuropneumonectomy in the management of diffuse malignant mesothelioma of the pleura. Experience with 29 patients. Thorax. 1976;31:15-24.

7. Sugarbaker DJ, Flores RM, Jaklitsch MT, Richards WG, Strauss GM, Corson JM, et al. Resection margins, extrapleural nodal status, and cell type determine postoperative long-term survival in trimodality therapy of malignant pleural mesothelioma: results in 183 patients. J Thorac Cardiovasc Surg. 1999;117:54-65.

8. Flores RM, Pass HI, Seshan VE, Dycoco J, Zakowski M, Carbone M, et al. Extrapleural pneumonectomy versus pleurectomy/decortication in the surgical management of malignant pleural mesothelioma: results in 663 patients. $J$ Thorac Cardiovasc Surg. 2008;135:620-6, e1-3.

9. Rice DC, Stevens CW, Correa AM, Vaporciyan AA, Tsao A, Forster KM, et al. Outcomes after extrapleural pneumonectomy and intensity-modulated radiation therapy for malignant pleural mesothelioma. Ann Thorac Surg. 2007;84:1685-93.

10. Richards WG, Zellos L, Bueno R, Jaklitsch MT, Janne PA, Chirieac LR, et al. Phase I to II study of pleurectomy/decortication and intraoperative intracavitary hyperthermic cisplatin lavage for mesothelioma. J Clin Oncol. 2006;24:1561-7.

11. Sugarbaker DJ, Jaklitsch MT, Bueno R, Richards W, Lukanich J, Mentzer SJ, et al. Prevention, early detection, and management of complications after 328 consecutive extrapleural pneumonectomies. J Thorac Cardiovasc Surg. 2004;128:138-46.

12. Flores RM, Krug LM, Rosenzweig KE, Venkatraman E, Vincent A, Heelan R, et al. Induction chemotherapy, extrapleural pneumonectomy, and postoperative high-dose radiotherapy for locally advanced malignant pleural mesothelioma: a phase II trial. J Thorac Oncol. 2006;1:289-95.

13. Pass HI, Temeck BK, Kranda K, Thomas G, Russo A, Smith P, et al. Phase III randomized trial of surgery with or without intraoperative photodynamic therapy and postoperative immunochemotherapy for malignant pleural mesothelioma. Ann Surg Oncol. 1997;4:628-33.

14. Baldini EH, Recht A, Strauss GM, DeCamp MM Jr, Swanson SJ, Liptay MJ, et al. Patterns of failure after trimodality therapy for malignant pleural mesothelioma. Ann Thorac Surg. 1997;63:334-8.

15. Gupta V, Mychalczak B, Krug L, Flores R, Bains M, Rusch VW, et al. Hemithoracic radiation therapy after pleurectomy/decortication for malignant pleural mesothelioma. Int J Radiat Oncol Biol Phys. 2005;63:1045-52.

16. Friedberg JS, Mick R, Stevenson J, Metz J, Zhu T, Buyske J, et al. A phase I study of Foscan-mediated photodynamic therapy and surgery in patients with mesothelioma. Ann Thorac Surg. 2003;75:952-9.
17. Chang MY, Sugarbaker DJ. Extrapleural pneumonectomy for diffuse malignant pleural mesothelioma: techniques and complications. Thorac Surg Clin. 2004; 14:523-30.

18. Zellos L, Richards WG, Capalbo L, Jaklitsch MT, Chirieac LR, Johnson BE, et al. A phase I study of extrapleural pneumonectomy and intracavitary intraoperative hyperthermic cisplatin with amifostine cytoprotection for malignant pleural mesothelioma. J Thorac Cardiovasc Surg. 2009;137:453-8.

19. Sugarbaker DJ, Mentzer SJ, Strauss G. Extrapleural pneumonectomy in the treatment of malignant pleural mesothelioma. Ann Thorac Surg. 1992;54:941-6.

20. Markman M, Cleary S, Howell SB. Nephrotoxicity of high-dose intracavitary cisplatin with intravenous thiosulfate protection. Eur J Cancer Clin Oncol. 1985;21: 1015-8.

21. Hensley ML, Schuchter LM, Lindley C, Meropol NJ, Cohen GI, Broder G, et al. American Society of Clinical Oncology clinical practice guidelines for the use of chemotherapy and radiotherapy protectants. J Clin Oncol. 1999;17:3333-55.

22. Sugarbaker DJ, Garcia JP, Richards WG, Harpole DH Jr, Healy-Baldini E, DeCamp MM Jr, et al. Extrapleural pneumonectomy in the multimodality therapy of malignant pleural mesothelioma. Results in 120 consecutive patients. Ann Surg. 1996;244:288-94.

23. Allen AM, Czerminska M, Janne PA, Sugarbaker DJ, Bueno R, Harris JR, et al. Fatal pneumonitis associated with intensity-modulated radiation therapy for mesothelioma. Int J Radiat Oncol Biol Phys. 2006;65:640-5.

24. Gray R. A class of K-sample tests for comparing the cumulative incidence of a competing risk. Ann Stat. 1988;16:1141-54.

25. Okada M, Mimura T, Ohbayashi C, Sakuma T, Soejima T, Tsubota N. Radical surgery for malignant pleural mesothelioma: results and prognosis. Interact Cardiovasc Thorac Surg. 2008;7:102-6.

26. Rusch VW, Venkatraman E. The importance of surgical staging in the treatment of malignant pleural mesothelioma. J Thorac Cardiovasc Surg. 1996;111:815-26.

27. Richards WG, Zellos L, Bueno R, Hartigan P, Sugarbaker DJ. Sequential amifostine and sodium thiosulfate improve renal protection during intracavitary high-dose cisplatin lavage. Presented at: 8th International Conference of the International Mesothelioma Interest Group; Chicago, Ill; 2006.

28. Ellis P, Davies AM, Evans WK, Haynes AE, Lloyd NS. The use of chemotherapy in patients with advanced malignant pleural mesothelioma: a systematic review and practice guideline. J Thorac Oncol. 2006;1:591-601.

29. van Meerbeeck JP, Gaafar R, Manegold C, Van Klaveren RJ, Van Marck EA, Vincent M, et al. Randomized phase III study of cisplatin with or without raltitrexed in patients with malignant pleural mesothelioma: an intergroup study of the European Organisation for Research and Treatment of Cancer Lung Cancer Group and the National Cancer Institute of Canada. J Clin Oncol. 2005;23: 6881-9.

30. Vogelzang NJ, Rusthoven JJ, Symanowski J, Denham C, Kaukel E, Ruffie P, et al. Phase III study of pemetrexed in combination with cisplatin versus cisplatin alone in patients with malignant pleural mesothelioma. J Clin Oncol. 2003;21:2636-44.

\section{Discussion}

Dr Valerie W. Rusch (New York, NY). Thank you for asking me to discuss this excellent article, which was extremely well presented.

During the past 20 years, there have been significant improvements in the management of this disease, including a better understanding of its biology, improved methods and staging, decreases in operative mortality, and the development of better radiation and chemotherapy treatments. However, MPM is still refractory to standard treatment approaches and is usually fatal. Therefore novel approaches to the treatment of this disease are clearly needed, and in this regard I congratulate your group for innovative work testing the application of hyperthermic intracavitary chemotherapy to patients undergoing resection for MPM.

Intracavitary chemotherapy in conjunction with maximal cytoreductive surgery has become a standard treatment option for peritoneal-based malignancies, such as metastatic ovarian cancer and primary peritoneal mesothelioma. The mortality and morbidity of this treatment strategy are clearly linked to the expertise of the 
surgical and anesthetic team because the operations are usually long and associated with high need for intravenous fluids and transfusions. Previous studies for peritoneal disease have shown that nonplatinum chemotherapy regimens appear to be associated with fewer serious adverse events, such as renal toxicity. Because intracavitary chemotherapy in either the pleural or peritoneal cavities penetrates to a depth of only about $5 \mathrm{~mm}$, successful treatment with this modality depends on the amount of tumor remaining after cytoreductive surgery. Moreover, multiple cycles of intraperitoneal chemotherapy or the addition of systemic therapy appear to play a role in achieving better long-term disease control.

Other studies have tested the application of intracavitary chemotherapy to malignant pleural effusions and to metastatic thymoma. In the early 1990s at Sloan-Kettering, we performed a phase II trial in patients with MPM who received intrapleural cisplatin and mitomycin without hyperthermia immediately after pleurectomy and decortication. Systemic chemotherapy was added postoperatively. Briefly, this trial showed that intrapleural chemotherapy can be administered safely by using standard measures of intensive hydration without renal protective agents but that the treatment approach was relatively ineffective in preventing local tumor recurrence. Importantly, pharmacokinetic studies from that trial showed that very high chemotherapy drug levels can be achieved intrapleurally but that systemic absorption was rapid, with peak plasma levels being reached within 1 hour, emphasizing the importance of protecting renal function when administering cisplatin intrapleurally.

The present study by the Brigham group and the 2 previous trials that they performed extend this experience with intrapleural chemotherapy in several ways: by adding hyperthermia, which is thought to enhance chemotherapy activity; by adding sodium thiosulfate and amifostine as renal protective agents to enable the use of very high-dose chemotherapy; and by perfusing both the pleural and peritoneal cavities in the hope of decreasing the risk of peritoneal, as well as pleural, disease recurrence.

As shown here, the combined modality treatment was feasible with a $4 \%$ in-hospital mortality but was associated with significant morbidity. In the manuscript draft that I received, there was an overall $14 \%$ risk of significant renal dysfunction and a $13 \%$ risk of deep venous thrombosis and pulmonary embolus, a problem that has also been noted with intraperitoneal chemotherapy.

Unfortunately, the median survivals are very similar to those observed in trials of other, simpler treatment strategies, such as resection and radiation, and appear less favorable than the median survivals in recently reported European and North American trials of induction systemic therapy followed by EPP and adjuvant hemithoracic radiation. Moreover, recurrence in the ipsilateral thorax and peritoneum with this approach remains quite frequent.

Therefore at this point, one could ask whether the treatment strategy used in this trial is ready for export into more routine clinical practice. I think the answer is no, given the substantial risk of treatment-related morbidity and mortality and median survivals that do not suggest superiority over other treatment regimens.
However, I hope that these provocative results will lead the Brigham group and other investigators to additional trials that will define the ultimate role of this approach in the treatment of this difficult disease. I would encourage them to consider pharmacokinetic studies that might identify ways to minimize renal toxicity. In this regard I would like to ask you 3 questions.

First, does the reduction of operative mortality from $11 \%$ in the first study reported by your group in the Journal of Clinical Oncology in which patients underwent pleurectomy and decortication to $4 \%$ in this trial that used EPP merely reflect a 10 -year difference in the patients' median age between those 2 studies, or is it related to other factors?

Dr Tilleman. Dr Rusch, thank you very much for the summary and the review comments.

There is a difference between those 2 studies, the one reported in the Journal of Clinical Oncology and the one we are presenting right now. The difference can be attributed to several things. One of them is, as you pointed out, the difference in patient age (71 years for the P/D strategy vs 60 years for the current EPP strategy). I would like to emphasize that in the Journal of Clinical Oncology we have reported $\mathrm{P} / \mathrm{D}$ in patients who were not eligible for EPP because of high risk; hence a higher mortality might be expected. The last contribution might be due to an improvement in the perioperative critical care during the years, and there is a learning curve here.

Dr Rusch. In a previous phase I trial you found that amifostine did not provide adequate protection against renal toxicity. Can you explain why it was added to thiosulfate in this study and why it appears that it might work?

Dr Tilleman. You are correct, the phase I study in which we applied only amifostine did not show significant renal protection; however, in this study we have shown that thiosulfate and amifostine administered together have created this change. In this study there were 9 patients who had renal toxicities with thiosulfate, and only a single patient had renal toxicity after the administration of amifostine in addition to thiosulfate. Therefore there might be a synergistic cytoprotective reaction, but as you quoted, amifostine alone did not provide such renal protection.

Dr Rusch. I will close with a third question. Given the frequency of intra-abdominal recurrence in this study and the well-known risk of tumor implantation by mesothelioma, have you reconsidered your strategy of perfusing both the chest and the abdomen?

Dr Tilleman. We reported in 1997 local recurrence within the ipsilateral hemithorax and by direct extension into the abdomen. ${ }^{14}$ Adding intraoperative intracavitary chemotherapy reduced local recurrences from $67 \%$ to $34 \%$, yet the abdominal recurrences did not change, even after adding intracavitary chemotherapy (Table 4).

Baldini and coworkers' work ${ }^{14}$ showed the same abdominal recurrence rate (50\% recurrence). Therefore, yes, a more rigorous treatment might be needed, including washing first the abdomen, omentectomy, and administration of systemic chemotherapy. All might reduce recurrences in the abdomen. 